'S P Sylvan, ${ }^{2} J$ Hedlund. 'Department of Medical Sciences, Uppsala Academic Hospital, Uppsala, Sweden; ${ }^{2}$ Department of Communicable Disease Control and Prevention, Uppsala County Council, Uppsala, Sweden

The study was conducted in 2004 and repeated in 2011 to compare the contact-tracing success rate of the partner notification services routinely provided by the community-based youth health centres in Uppsala County, Sweden.

Objective The study had two goals, (i) to compare the number of sexual partners routinely reported by each diagnosed index case with CT and the success rate in tracing and testing these partners for CT infection in 2004 and 2011 (ii) To compare the notification practises in reporting the number of cases of unsuccessful contact tracing to the CMO in 2004 and 2011.

Successful contact-tracing is defined as the confirmed attendance of a sexual contact within 12 months of the contact with the index case.

Results The number of CT cases diagnosed by the youth health centres during 2004 was 463 (299 females/164 males) and 529 (339/190) during 2011. The females reported 660 male sexual contacts 2004 and 861 in 2011 and the males reported 386 female contacts in 2004 and 494 in 2011. Successful partner notification was achieved for $73 \%$ of all sexual contacts in 2004 but only $64 \%$ in 2011. 164 (74 females and 90 males) unsuccessful partner notifications were reported to the $\mathrm{CMO}$ in 2004 and the corresponding numbers for 2011 were 288 (105 females and 183 males).

Conclusions Between 2004 and 2011 there has been an increase in the mean number of sexual contacts among young adults in Uppsala County. Successful partner notification was significantly reduced. When asymptomatic, genital CT infection spreads among sexually active young adults with multiple, unidentified sexual partners, appropriate methods of partner notification are not sufficient to achieve its aims at the population level. Novel strategies are needed.

\section{P3.380 BASELINE RESULTS OF A CLUSTER-RANDOMISED TRIAL ASSESSING THE EFFECTIVENESS OF SPORT-BASED HIV PREVENTION IN SOUTH AFRICAN SCHOOLS}

doi:10.1136/sextrans-2013-051184.0833

1,2Z A Kaufman, 'E B Kaufman, 'S Dringus, ' H A Weiss, ${ }^{2}$ S Delany-Moretlwe, 'D A Ross 'London School of Hygiene and Tropical Medicine, London, UK; 'Wits Reproductive Health and HIV Institute, Johannesburg, South Africa

Background HIV prevalence among 15-24 year-olds in South Africa is amongst the highest in the world. A cluster-randomised trial began in 2012, assessing the effectiveness of a sport-based HIV prevention intervention in reducing age-disparate partnerships, multiple partnerships, and perpetration of gender-based violence.

Methods Forty-six schools in Cape Town and Port Elizabeth were randomised to either receive sessions led by trained Grassroot Soccer Coaches or standard Life-Orientation classes led by teachers. At baseline, 4485 grade-nine students (median age 15) used touchscreen mobile phones to complete a self-administered questionnaire assessing demographic/socio-economic, psychosocial, lifestyle, behavioural and relationship factors. Answers were confidential, with no personal identifying information captured. Chi-squares were used to assess crude differences by group, followed by multivariate linear and logistic regressions adjusting for age and schoollevel clustering.

Results The control group was slightly older than the intervention group (mean age 15.8 vs 15.5 years, $p=0.071$ ). At baseline, more control than intervention participants reported not living with both parents $(67.8 \%$ vs $64.1 \%, p=0.009)$, ever having had sex $(55.9 \%$ vs $52.6 \%, \mathrm{p}=0.032)$, having an age-disparate partner $(5+$ years difference) in the last year $(14.5 \%$ vs $12.1 \%, \mathrm{p}=0.097)$, depressive symptoms $(28.7 \%$ vs $25.7 \%, p=0.024)$, and HIV stigma (27.4\% vs
$23.8 \%, p=0.007)$. More males in the control group reported ever having perpetrated intimate-partner violence $(37.6 \%$ vs $33.4 \%$, $p=0.051)$ and rape $(29.1 \%$ vs $23.7 \%, p=0.029)$. These differences between trial arms lost statistical significance after adjusting for age and clustering. No differences between groups were observed for sex, socio-economic status, HIV knowledge, self-efficacy, or number of sexual partners.

Conclusions There was a small age imbalance between study groups, which appears to explain other observed baseline imbalances. Outcome analyses for the main trial should be adjusted for age. High levels of reported intimate-partner violence and rape perpetration were observed, suggesting this is a crucial area for intervention

\section{P3.381 KNOWLEDGE AND ATTITUDE TOWARDS HIV/AIDS AMONG POST-SECONDARY SCHOOL MALAYSIAN URBAN YOUTHS}

doi:10.1136/sextrans-2013-051184.0834

'K Shamsuddin, ${ }^{2} \mathrm{M}$ Ghafari, 'R Mohd Amin, 'R Sutan, 'S E Wan Puteh, 'S A Shah, ${ }^{3} \mathrm{Z}$ A Mahdy, ${ }^{3}$ S Ahmad. 'Dept of Community Health, Universiti Kebangsaan Malaysia Medical Centre, Kuala Lumpur, Malaysia; '2Dept of Public Health, Faculty of Health, Shahre Kord University of Medical Science, Shahre Kord, Iran; ${ }^{3}$ Dept of Obstetrics and Gynaecology, Universiti Kebangsaan Malaysia Medical Centre, Kuala Lumpur, Malaysia

Background Post secondary school, young people may continue to study or will seek work opportunities. They are exposed to a different environment which may influence their knowledge and attitude towards HIV/AIDS. The aim of this paper is to compare the knowledge and attitude of post-secondary school urban youths towards HIV/AIDS

Methods This was a cross-sectional survey using self administered questionnaire of young urban Malaysians age 18-24 years who were either enrolled in randomly selected private or public colleges and universities, or formally employed in private or public workplaces in the Klang Valley.

Results Compared to the 973 students, the 615 workers who participated in this study were significantly older $(22.6+1.5$ years versus $20.4+1.7$ years), $12.2 \%$ versus $0.8 \%$ were married, and $88.5 \%$ versus $80.1 \%$ were Muslims. Both groups had fairly good knowledge of HIV/AIDS and its prevention and did not show significant difference in their mean knowledge of HIV/AIDS. However, misconception on transmission of the disease persist when $35-40 \%$ thought sitting on toilet seats and $50 \%$ thought HIV can be transmitted through mosquito bites; and $47.5 \%$ of workers and $51.3 \%$ of students had negative attitudes towards HIV and people with HIV/ AIDS. Only $48.1 \%$ of workers and $41.7 \%$ of students were sure that condom is an effective way of protecting against HIV/AIDS while both, about $20 \%$, reported peers, and $18.0 \%$ students and $9.8 \%$ workers reported teachers/school counsellors as important sources of information on HIV/AIDS.

Conclusion Knowledge and attitude of the two groups of youths did not show significant differences although working youths seems to be slightly more open about HIV/AIDS and the use of condom for prevention of STDs. Workplace or college-based health promotion for prevention of HIV/STDs should be developed and maintained since misconception remains and negative attitude is still prevalent in these post-secondary school youths.

\section{P3.382 POST-NEONATAL PEDIATRIC CIRCUMCISION IN THE UNITED STATES, 2010}

doi:10.1136/sextrans-2013-051184.0835

'G D Hart-Cooper, 'G Tao, ${ }^{2} \mathrm{~J}$ Stock, ${ }^{1} \mathrm{~K}$ Hoover. ${ }^{1}$ Centers for Disease Control and Prevention, Atlanta, GA, United States, ${ }^{2}$ Mount Sinai School of Medicine, New York, NY, United States 
Background Male circumcision can protect against sexually transmitted infections, HIV, and urinary tract infections. The procedure is easier to perform during the neonatal period ( $<28$ days), with fewer complications and rarely requiring general anaesthesia. Few studies have estimated the number of circumcisions, or indications for the procedure, in the post-neonatal period (ages 1-18 years). Our objective was to compare these for neonatal and post-neonatal circumcisions.

Methods We analysed MarketScan data, a database of billing claims from commercial health plans. We used circumcision procedural codes to identify all circumcisions in 2010, including neonatal circumcisions of males born to women enrolled in the health plans, and circumcisions of males aged 1-18 years. We assessed reasons for circumcision using diagnostic codes, and stratified the number of circumcisions and associated diagnosis by age. We estimated the neonatal circumcision rate.

Results Overall, 120,994 circumcisions were performed in 2010, with $113,740(94 \%)$ in neonates and 7,254 (6.0\%) in post-neonates. Among post-neonatal circumcisions, $67 \%$ were performed for boys $<3$ years of age and of these $28 \%$ were elective. In contrast, among males 3 years and older, only $8 \%$ were elective. The neonatal circumcision rate was $113,740 / 182,503$ (62\%), and 92\% were elective. Among 16,457 non-elective circumcisions for both neonates and post-neonates, the most frequent indications were phimosis $(92 \%)$, balanitis (3\%), hidden penis $(2 \%)$, chordee $(2 \%)$, and hypospadias $(2 \%)$.

Conclusion Most post-neonatal circumcisions were performed among males $<3$ years, and were 8.6 times higher than circumcisions among males 3 years and older. The large number of elective post-neonatal circumcisions in males $<3$ years suggest that neonatal circumcision might be a missed opportunity for these boys. Delaying elective circumcision results in greater risk for the child, and a more costly procedure. Discussions with parents early in pregnancy might help them make an informed decision about circumcision of their child.

\section{P3.383 MAPPING AND SIZE ESTIMATION OF VULNERABLE YOUTH IN THE WESTERN PROVINCE, SRI LANKA}

doi:10.1136/sextrans-2013-051184.0836

\section{J P h Vidanapathirana, A d Karavita. National STD/AIDS Control Programme,} COLOMBO 10, Sri Lanka

Background All youth in Sri Lanka are not at risk of HIV/AIDS. However, certain socio-economic and behaviour factors are present among youth which make them vulnerable. There are subsets of especially vulnerable youth (EVY) and most-at-risk youth who need to be identified for targeted interventions. Identification of types of subsets and size are essential for interventions. The Objective of this is to identify the subsets of EVY and estimate the size according to the geographical area in the Western Province,

Method Groups of EVY in the western province were identified using participatory approach with broad based consultation of various stakeholders in the public, private and civil society organisation including youth organisations.

Geographic mapping and size estimation was done using the participatory method in group consensus with the key partners. Previous studies, ad hoc surveys, programmatic data from the various organisations including census and statistic data, and experience of the participants were used.

Series of mapping workshops conducted to finalise the locations and size of the population groups identified as EVY. This was done interactively within each group of experts assigned to each study zone. Locations were marked as a point layer manually on Topographic maps. Each point was assigned a definite number relevant to the size.
Results Ten number groups of EVY were identified with total of 47,285 in the Western Province. Final results were generated by using the topographic maps of the Western Province. These maps were then scrutinised carefully in a systematic way to read the locations and the number of the population group marked by group ID. Different point layers for each identified EVY was created on digital topographic maps.

Conclusion Ten groups of EVY were identified according to the size and geographical areas. These data will be using future HIV prevention interventions with different approaches.

\section{P3.384 ESTIMATING THE IMPACT OF COMBINED PREVENTION INTERVENTIONS TARGETING 15-24 YEARS-OLD MEN AND WOMEN IN NYANZA, KENYA}

doi:10.1136/sextrans-2013-051184.0837

'R Alsallaq, ${ }^{1} \mathrm{~J}$ Buttolph, ${ }^{1} \mathrm{C}$ Cleland, ${ }^{2} \mathrm{~T}$ Hallett, 'A Kurth. 'College of Nursing Global, New York University, New York, NY, United States; ${ }^{2}$ mmperial College, London, UK

Background Young males (YMs) and especially young females (YFs) (age 15-24) in sub-Saharan Africa are at a higher risk of HIV infection compared to older adults. HIV testing of young individuals facilitates early identification of their 'HIV +' status, prompt ART initiation, and provision of male circumcision and PrEP. We hypothesise that youth-prioritised combination interventions could have substantial impact on HIV incidence among them and the wider adult population.

Methods We constructed a mathematical model that represented HIV heterosexual transmission in Nyanza, Kenya and used local data to specify cross-generational sex, risk- and age-dependent behaviours, and school attendance. We estimated the impact of leveraging HIV testing and counselling for condom use among Nyanza youth, prompt ART initiation (at CD $4 \leq 350$ cells $/ \mathrm{mm}^{3}$ ) for those newly-found infected, and gender-specific interventions for YMs and YFs. The former reaching $80 \%$ circumcision among ' $\mathrm{HIV}$-' YMs while the later reaching 40\% PrEP coverage among 'HIV-' YFs not attending school and halving the proportion of partnerships that YFs attending school form with $20+$ years-old men.

Results We predict a reduction in HIV incidence over 10 years among youth by $38 \%$ (from $1.6 \%$ /person-years) and adults by $29 \%$ (from 1.2\%/person-years), if the annual testing likelihood for youth increases to $90 \%$ with those newly-found infected increasing condom use by $30 \%$ and initiating ART promptly. The adult incidence is reduced $34 \%$ and $35 \%$ by further male and female specific interventions when applied separately. The full package with all interventions combined would decrease incidence among youth and adults by $59 \%$ and $40 \%$, respectively, and reduce the lifetime HIV risk experienced by YFs by $24 \%$.

Conclusion In populations where young people are at the highest risk of HIV infection, carefully prioritised, gender-specific intervention can have a substantial impact on the risk of infection, both in that group and the overall population.

\section{P3.385 COMPARATIVE EXPERIENCE AND OUTCOMES OF CLINIC STAFF VERSUS INTENSIVE RESEARCHER LED RECRUITMENT TO A SEXUAL HEALTH INTERVENTION IN UK PRIMARY CARE}

doi:10.1136/sextrans-2013-051184.0838

1J A Cassell, ${ }^{2} \mathrm{~J}$ Dodds, ${ }^{1} \mathrm{~S}$ Lanza, ${ }^{3} \mathrm{~N}$ Low, ${ }^{4} \mathrm{~T}$ Roberts, ${ }^{1} \mathrm{H}$ E Smith, ${ }^{5} \mathrm{G}$ Rait. ${ }^{1}$ Brighton and Sussex Medical School, Brighton, UK; '²ueen Mary, University of London, London, UK; ${ }^{3}$ University of Bern, Bern, Switzerland; ${ }^{4}$ University of Birmingham, Birmingham, UK; ${ }^{5}$ University College London, London, UK

Background As part of a national randomised controlled trial, we attempted to recruit young people for Chlamydia testing and 